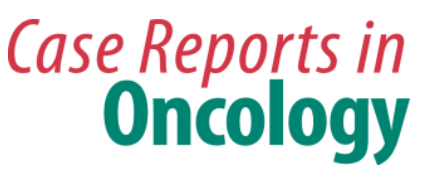

Case Rep Oncol 2017;10:726-731

DOI: $10.1159 / 000479499$

Published online: August 9, 2017
(C) 2017 The Author(s)

Published by S. Karger AG, Basel www.karger.com/cro

This article is licensed under the Creative Commons Attribution-NonCommercial 4.0 International License (CC BY-NC) (http://www.karger.com/Services/OpenAccessLicense). Usage and distribution for commercial purposes requires written permission.

\title{
Targeted Approaches Applied to Uncommon Diseases: A Case of Salivary Duct Carcinoma Metastatic to the Brain Treated with the Multikinase Inhibitor Neratinib
}

\author{
Karl R. Sorenson ${ }^{a} \quad$ Guilherme Piovezani Ramos ${ }^{a}$ \\ Jose Caetano Villasboas Bisneto ${ }^{c} \quad K^{2}$ atharine Price ${ }^{b}$ \\ ${ }^{a}$ Department of Internal Medicine, Mayo Clinic, Rochester, MN, USA; ${ }^{b}$ Department of \\ Oncology, Division of Medical Oncology, Mayo Clinic, Rochester, MN, USA; ${ }^{c}$ Department \\ of Medicine, Division of Hematology, Mayo Clinic, Rochester, MN, USA
}

\section{Keywords}

Salivary duct carcinoma - Intracranial metastasis - Neratinib · Human epidermal growth factor receptor $2 \cdot$ Targeted therapy

\begin{abstract}
Salivary duct carcinoma is a rare malignancy associated with hormone receptor and human epidermal growth factor receptor 2 (HER2) overexpression. Local surgical control is the cornerstone of therapy, but a subset of patients develops metastatic disease portending a poor prognosis and limited management options. Intracranial metastases are an uncommon manifestation and present a therapeutic challenge. We report the case of a 31-year-old male who presented with facial pain and swelling subsequently diagnosed with salivary duct carcinoma. Our patient underwent extensive locoregional resection and analysis of the tumor tissue demonstrated evidence of androgen receptor expression and HER2 overexpression. His course was complicated by metastatic extra- and intracranial recurrence despite combined modality treatment with radiation and chemotherapy followed by anti-HER2 monoclonal antibody therapy and androgen deprivation therapy. After exhausting standard treatment options, he received experimental therapy with a new small-molecule tyrosine kinase inhibi-
\end{abstract}


tor, neratinib, with evidence of a transient clinical response and no significant adverse effects. This case exemplifies the potential and limitations of targeted therapy, particularly when applied to patients with rare diseases and presentations.

(C) 2017 The Author(s)

Published by S. Karger AG, Basel

\section{Introduction}

Salivary duct carcinoma (SDC) is a rare, aggressive type of salivary cancer with a high potential for distant metastases [1]. The majority of SDC express the androgen receptor with variable human epidermal growth factor receptor 2 (HER2) amplification [2-6]. Responses to both androgen blockade and anti-HER2 therapies have been reported [7]. Brain metastases are uncommon but represent a treatment challenge given the poor intracranial penetration of many drugs. Neratinib (HKI-272) is a potent, oral, irreversible inhibitor of the EerbB family of receptor tyrosine kinases that inhibits signal transduction through ErbB1 (endothelial growth factor receptor), ErbB2 (HER2), and ErbB4. Emerging clinical data has shown evidence of brain penetration of neratinib, and neratinib is currently under investigation alone or with chemotherapy for HER2-positive breast cancer with brain metastases (NCT01494662) and other cancers [8, 9]. Here, we describe a patient with refractory metastatic SDC with progressive cerebellar metastases who obtained a transient clinical benefit from treatment with neratinib.

\section{Case Presentation}

In November 2007, a 31-year-old male presented with progressive right-sided facial swelling and pain. On July 9, 2008, a right superficial parotidectomy was performed, revealing a 1.8-cm (at least T1 per AJCC TNM), high-grade SDC with positive margins. Five of 5 regional lymph nodes were involved with metastatic disease. A positron emission tomography-computed tomography (PET-CT) scan obtained on July 15, 2008, revealed postoperative changes within the right parotid bed and persistent adenopathy within the right parotid bed and right neck but no evidence of distant metastatic disease. Seven days later, the patient was taken back to the operating room and underwent a right modified neck dissection with 15 of 16 level II, 10 of 11 level III, and 13 of 13 level IV lymph nodes positive for metastatic SDC with extracapsular extension (at least N2b per AJCC TNM). The initial stage was consistent with stage IVA (T1 N2b M0) according to AJCC TNM.

From August 2008 through October 2008, the patient received adjuvant radiation therapy (70 Gy in 35 fractions) with concomitant high-dose cisplatin. In May 2009, the patient was found to have asymptomatic bilateral pulmonary nodules and was started on the antiHER2 monoclonal antibody trastuzumab with complete resolution of the pulmonary nodules on subsequent imaging. The patient remained on trastuzumab without evidence of systemic disease for nearly 3 years.

In March 2012, he presented with ataxia and was found to have a solitary right cerebellar lesion on brain magnetic resonance imaging (MRI). No evidence of extracranial FDG-avid disease was found on PET-CT. On March 12, 2012, the patient underwent craniotomy and resection of the cerebellar mass. Pathology was consistent with metastatic SDC with overexpression of HER2 and androgen receptor by immunohistochemistry along with confirmed HER2 gene amplification by FISH (HER2:D17Z1 ratio of 10.3). He subsequently received adjuvant radiation therapy to the tumor bed ( 5 Gy in 5 fractions), and trastuzumab was rei- 
nitiated. Several months later, lapatinib was added to trastuzumab. MRI on January 31, 2013, showed new nodularity at the tumor bed concerning for recurrence, which was treated with salvage stereotactic radiosurgery. In June 2013, a new lesion at the cerebellar vermis was noted, and the patient again underwent salvage radiosurgery. Trastuzumab was stopped, but lapatinib was continued in combination with capecitabine. Six months later - in January 2014 - MRI again documented tumor recurrence in the right cerebellum, and both capecitabine and lapatinib were stopped. PET-CT continued to show no evidence of extracranial disease. On January 28, 2014, the patient underwent salvage resection of recurrent tumor with pathology again showing SDC. In February 2014, treatment with trastuzumab emtansine (Kadcyla ${ }^{\circledR}$ or TDM-1) was started and he received 6 cycles, but due to insurance coverage issues it was subsequently stopped. At this point, the patient presented to Mayo Clinic in Rochester, MN, USA, for a second opinion. As he was hormone therapy-naïve, he was initiated first on combined androgen blockade with Lupron and bicalutamide with disease progression in the cerebellum followed by second-line hormone therapy with abiraterone and steroids, also with progressive intracranial disease. MRI on December 18, 2014, showed increasing cerebellar metastases with a mass in the right cerebellar peduncle measuring 3.3 $\mathrm{cm}$ and a vermis mass measuring $2.1 \mathrm{~cm}$ with increased reactive edema (Fig. 1a). On December 31, 2014, the patient initiated neratinib therapy through an emergency use singlepatient protocol. Neratinib was tolerated well without adverse events. Prophylactic loperamide was used during the first cycle. Clinically, the patient noted improvement in symptoms with decreased headache and ataxia. Follow-up imaging 1 month later showed stable findings, and neratinib therapy was continued. Repeat imaging on March 16, 2015, showed mild disease progression in the cerebellum, and oral cyclophosphamide was added to try and enhance treatment response (Fig. 1b). In the subsequent weeks, the patient developed gradual progression of fatigue, diplopia, and decline in performance status. In early May 2015 , due to escalating symptom burden, the patient was transitioned to hospice care, eventually passing away on May 21, 2015.

\section{Discussion}

This case outlines the advances in personalized adaptive treatment options in oncology in patients with rare cancers and atypical presentations. It also underscores the need to improve our understanding regarding optimal targets, sequences, and combinations of such therapies.

SDC is a rare malignancy with historically poor outcomes. The median overall survival ranged from 37 to 79 months $[10,11]$. The majority of patients present with advanced locoregional disease of the parotid gland, and the cornerstone of management is radical surgical resection with or without radiation. However, distant treatment failure is seen in 39$52 \%$ of the patients, necessitating systemic treatment $[11,12]$. The most common site of distant metastases is the lung, with central nervous system (CNS) involvement representing a minority of patients (5\%). There is a paucity of data regarding systemic management of these patients, particularly with CNS recurrence as in the present case.

There have been emerging efforts to translate advances from other tumor types into SDC treatment options. SDC has histopathologic similarities to ductal breast carcinomas, with a varying reported incidence of HER2 expression (25-83\%) [2-6]. In addition, most of these tumors express androgen receptors (44-100\%) [4]. Targeted therapies with HER2 inhibition and androgen deprivation therapy have resulted in objective responses as single 


\section{Case Reports in Oncology}

agents or in combination with chemotherapy $[7,13]$. However, many of these agents have either limited (e.g., trastuzumab, docetaxel) or unknown (e.g., androgen deprivation therapy) activity in the CNS. To our knowledge, this is the first report of the use of the multitargeted tyrosine kinase inhibitor (TKI) neratinib for HER2 positive SDC metastatic to the brain.

HER2 is a membrane-bound tyrosine kinase receptor with both extracellular and intracellular domains whose activation results in downstream signaling cascades that support cell survival and proliferation. Therapeutic agents have been developed targeting the extracellular domain (trastuzumab, pertuzumab) in addition to emerging small-molecule TKIs (smTKIs) designed to block the intracellular activation of this receptor (e.g., lapatinib, neratinib). Some of the newer smTKIs are also aimed to exploit characteristics unique to the blood-brain and blood-tumor barrier with the goal of targeting CNS disease. This experience has been made with lapatinib, which has shown both single-agent and combined CNS activity in metastatic breast cancer. While the dose response is unclear, lapatinib has shown relatively low CNS concentrations attributed in part to drug efflux, potentially limiting its efficacy in CNS disease [14-16].

Neratinib is an oral, irreversible, smTKI of HER1, HER2, and HER4 that targets intracellular phosphorylation interfering with receptor dimerization and downstream signaling. The molecule exhibits similar favorable characteristics shared by other smTKIs that favor CNS delivery (small molecule, lipophilic) but has also shown in vitro chemoresistance to several CNS efflux transporters, posing a theoretical mechanism to attain higher CNS concentrations $[16,17]$. Neratinib has demonstrated peripheral activity in breast cancer as a single agent and in combination therapy in addition to phase II data suggesting CNS activity in the management of metastatic brain lesions $[8,18-21]$. The choice of neratinib as experimental therapy in this patient was based on the combination of its specific molecular action on the HER2 receptor and its potential to penetrate the CNS.

Our patient received systemic treatment with chemotherapy, hormonal therapy, and targeted therapy, taking advantage of tumor-specific pathways with treatments adapted from more common tumor subtypes using molecularly driven treatment options. He attained a durable response from targeted therapy in the setting of peripheral metastatic disease, unfortunately complicated by CNS relapse, which represents a growing clinical challenge in oncology. He was able to achieve a transient clinical benefit from CNS-directed therapy, including the novel use of neratinib as a receptor-specific targeted therapy for brain metastases in SDC. It remains unclear how prior treatments influence the use of neratinib or whether therapeutic benefit would be increased if neratinib had been used in combination with other agents or earlier in his disease course. It is important to note that this patient did not experience any significant adverse effects from therapy with neratinib despite multiple prior treatment lines. There is certainly a critical need for additional scientific information on the use of this drug in patients who harbor overexpression of the HER2 receptor.

\section{Conclusion}

Neratinib is a novel smTKI targeting the HER2 pathway that has shown activity in metastatic CNS disease. To our knowledge, this is the first case of the use of neratinib in SDC with brain metastases. Treatment provided a transient clinical response suggesting a degree of CNS activity in the salvage setting after multiple lines of therapy without significant adverse effects. 
This case highlights several important aspects of ongoing cancer management. First, there are limited data on systemic management targeting metastatic disease to the brain, particularly with targeted therapies. Second, emerging hormonal, molecular, and genetic tumor analysis has provided an array of new treatment strategies; however, the sequence and combination of these therapies remains largely understudied. Third, it reinforces the continued need for adaptive trial designs to take advantage of such therapies in uncommon malignancies to help improve outcomes being mindful of cost-effective care.

\section{Teaching Points}

1 SDC is a rare malignancy associated with androgen receptor and HER2 pathway overexpression with limited effective systemic treatment options.

2 Our understanding of tumor biology has created a growing therapeutic environment necessitating coordination and adaptation of basic science and clinical experience to provide advances in understudied areas of oncologic care.

3 Isolated intracranial metastasis presents a unique therapeutic challenge with regards to systemic treatment options, remaining largely understudied, particularly in the era of targeted agents.

4 Neratinib is a smTKI that may provide a therapeutic option for the treatment of CNS disease in malignancies with HER2 overexpression, although its role in the management of SDC remains largely exploratory.

\section{Statement of Ethics}

The authors have no ethical conflicts to disclose.

\section{Disclosure Statement}

The authors have no conflicts of interest to disclose.

\section{References}

1 Guzzo M, Di Palma S, Grandi C, Molinari R: Salivary duct carcinoma: clinical characteristics and treatment strategies. Head Neck 1997;19:126-133.

-2 Johnson CJ, Barry MB, Vasef MA, Deyoung BR: Her-2/neu expression in salivary duct carcinoma: an immunohistochemical and chromogenic in situ hybridization study. Appl Immunohistochem Mol Morphol 2008;16:54-58.

- 3 Clauditz TS, Reiff M, Gravert L, Gnoss A, Tsourlakis MC, Munscher A, et al: Human epidermal growth factor receptor 2 (HER2) in salivary gland carcinomas. Pathology 2011;43:459-464.

4 Williams MD, Roberts D, Blumenschein GR Jr, Temam S, Kies MS, Rosenthal DI, et al: Differential expression of hormonal and growth factor receptors in salivary duct carcinomas: biologic significance and potential role in therapeutic stratification of patients. Am J Surg Pathol 2007;31:1645-1652.

-5 Williams MD, Roberts DB, Kies MS, Mao L, Weber RS, El-Naggar AK: Genetic and expression analysis of HER-2 and EGFR genes in salivary duct carcinoma: empirical and therapeutic significance. Clin Cancer Res 2010;16:2266-2274.

6 Negri T, Tamborini E, Dagrada GP, Greco A, Staurengo S, Guzzo M, et al: TRK-A, HER-2/neu, and KIT expression/activation profiles in salivary gland carcinoma. Transl Oncol 2008;1:121-128.

7 Alotaibi AM, Alqarni MA, Alnobi A, Tarakji B: Human epidermal growth factor receptor 2 (HER2/neu) in salivary gland carcinomas: a review of literature. J Clin Diagn Res 2015;9:ZE04-ZE08. 


\section{Case Reports in Oncology}

\begin{tabular}{l|l}
\hline Case Rep Oncol 2017;10:726-731 \\
\hline DOI: $10.1159 / 000479499$ & $\begin{array}{l}\text { (c) 2017 The Author(s). Published by S. Karger AG, Basel } \\
\text { www.karger.com/cro }\end{array}$ \\
\hline
\end{tabular}

Sorenson et al: Targeted Approaches Applied to Uncommon Diseases: A Case of Salivary Duct Carcinoma Metastatic to the Brain Treated with the Multikinase Inhibitor Neratinib

8 Chan A, Delaloge S, Holmes FA, Moy B, Iwata H, Harvey VJ, et al: Neratinib after trastuzumab-based adjuvant therapy in patients with HER2-positive breast cancer (ExteNET): a multicentre, randomised, double-blind, placebo-controlled, phase 3 trial. Lancet Oncol 2016;17:367-377.

-9 Bose P, Ozer H: Neratinib: an oral, irreversible dual EGFR/HER2 inhibitor for breast and non-small cell lung cancer. Expert Opin Investig Drugs 2009;18:1735-1751.

10 Gilbert MR, Sharma A, Schmitt NC, Johnson JT, Ferris RL, Duvvuri U, et al: A 20-year review of 75 cases of salivary duct carcinoma. JAMA Otolaryngol Head Neck Surg 2016;142:489-495.

-11 Johnston ML, Huang SH, Waldron JN, Atenafu EG, Chan K, Cummings BJ, et al: Salivary duct carcinoma: treatment, outcomes, and patterns of failure. Head Neck 2016;38(suppl 1):E820-E826.

12 Otsuka K, Imanishi Y, Tada Y, Kawakita D, Kano S, Tsukahara K, et al: Clinical outcomes and prognostic factors for salivary duct carcinoma: a multi-institutional analysis of 141 patients. Ann Surg Oncol 2016;23:2038-2045.

13 Lagha A, Chraiet N, Ayadi M, Krimi S, Allani B, Rifi H, et al: Systemic therapy in the management of metastatic or advanced salivary gland cancers. Oral Oncol 2012;48:948-957.

14 Chien AJ, Rugo HS: Emerging treatment options for the management of brain metastases in patients with HER2-positive metastatic breast cancer. Breast Cancer Res Treat 2013;137:1-12.

15 Saleem A, Searle GE, Kenny LM, Huiban M, Kozlowski K, Waldman AD, et al: Lapatinib access into normal brain and brain metastases in patients with Her-2 overexpressing breast cancer. EJNMMI Res 2015;5:30.

-16 Sanchez-Martin M, Pandiella A: Differential action of small molecule HER kinase inhibitors on receptor heterodimerization: therapeutic implications. Int J Cancer 2012;131:244-252.

-17 Feldinger K, Kong A: Profile of neratinib and its potential in the treatment of breast cancer. Breast Cancer (Dove Med Press) 2015;7:147-162.

-18 Chan A: Neratinib in HER-2-positive breast cancer: results to date and clinical usefulness. Ther Adv Med Oncol 2016;8:339-350.

19 Burstein HJ, Sun Y, Dirix LY, Jiang Z, Paridaens R, Tan AR, et al: Neratinib, an irreversible ErbB receptor tyrosine kinase inhibitor, in patients with advanced ErbB2-positive breast cancer. J Clin Oncol 2010;28:1301-1307.

20 Park JW, Liu MC, Yee D, Yau C, van 't Veer LJ, Symmans WF, et al: Adaptive randomization of neratinib in early breast cancer. N Engl J Med 2016;375:11-22.

-21 Freedman RA, Gelman RS, Wefel JS, Melisko ME, Hess KR, Connolly RM, et al: Translational Breast Cancer Research Consortium (TBCRC) 022: a phase II trial of neratinib for patients with human epidermal growth factor receptor 2-positive breast cancer and brain metastases. J Clin Oncol 2016;34:945-952.


Fig. 1. a Magnetic resonance imaging (MRI) axial T2-weighted fluid-attenuated inversion recovery image prior to initiation of neratinib. b MRI axial T2-weighted fluid-attenuated inversion recovery image 3 weeks after initiation of neratinib. 